\title{
Die Bedeutung der Sprecheridentität für die Zuschreibung von „Normalität“ bei Kommunikationsstörungen
}

\author{
Christiane Neuschaefer-Rube und Dominik Groß
}

\section{Einleitung}

Die verbale Kommunikationsfähigkeit des Menschen gilt als besonderer Ausdruck seiner biologischen und kulturellen Entwicklung, verbinden sich doch im übermittelten Sprachsignal stimmliche, sprecherische und sprachliche Merkmale einer Person zu einem komplexen Gebilde einer individuellen Sprecheridentität, der im Allgemeinen ein ähnlich hoher personaler Identifizierungscharakter ${ }^{1}$ zugesprochen wird wie dem menschlichen Gesicht. So beinhaltet das übermittelte Sprachschallsignal ${ }^{2}$ neben den sprachlichen Inhalten auch Informationen über Alter, Geschlecht, ${ }^{3}$ Bildungsgrad, Peer-Gruppenzugehörigkeit des jeweiligen Sprechers ebenso wie über dessen aktuelle psychische Befindlichkeit, aber auch über die emotionale Bewertung ${ }^{4}$ der von ihm geschilderten konzeptuellen, personenbezogenen oder situativen Inhalte. Die Gesamtheit der im Sprachschall repräsentierten Merkmale ermöglicht es, den Sprecher unabhängig vom visuellen Kontakt als eine bestimmte Person zu identifizieren, vorausgesetzt, Sprecher und Empfänger des Kommunikationsgeschehens sind miteinander bekannt. Verwechslungen besonders naher Angehöriger sind zwar möglich, widersprechen dem Konzept der sprachschallgebundenen Identifizierungsmöglichkeit von Personen jedoch keineswegs, da

\footnotetext{
1 Vgl. Stengel/Strauch (1996).

2 Vgl. Baken/Orlikoff (2000).

3 Vgl. Titze (1989) sowie Neuschaefer-Rube/Scheidt/Groß in diesem Band.

4 Vgl. Spiecker-Henke (1997), S. 12 und Neuschaefer-Rube/Spiecker-Henke (2002), S. 283.
} 
die verwechselten Personen typischerweise phonatorische und/oder verbale Eigenschaften aufweisen, die durchaus als gemeinsame Identitätsmerkmale aufgefasst werden können.

Zahlreiche Formen von fortgeschrittenen Stimm-, Sprech- und Sprachstörungen gehen mit einem temporären oder dauerhaften Verlust dieser Sprecheridentität einher - ein Verlust, der von den Betroffenen und ihren unmittelbaren Bezugspersonen als wesentliche Einschränkung der Lebensqualität bewertet wird. Wenn Personen, die an derartigen Störungen erkrankt sind, häufig berichten, sie würden sich selbst stimmlich und sprachlich nicht mehr erkennen, ist ihnen oft nicht klar, ob ihre reduzierte Sprechverständlichkeit oder ihr erlebter Identitätsverlust den stärkeren Leidensdruck ausüben. Des ungeachtet wirken sich Einschränkungen oder sogar eine vollständige Einbuße dieser identitätsstiftenden Eigenschaften und Fähigkeiten auf die betroffenen Personen häufig derart belastend aus, dass ein (sprachliches) Vermeidungsverhalten bis zu einem psychosozialen Rückzug häufige Folge ist.

Noch stärker als der Identitätsverlust können ursachenbezogene Fehldeutungen belasten, die den beeinträchtigten Sprecher sozial ausgrenzen. Ein bislang wenig diskutiertes, aber typisches Beispiel hierfür ist die Missinterpretation einer bestehenden Dysarthrie als Sprechen im alkoholisierten Zustand oder die fälschliche Zuschreibung reduzierter intellektueller Fähigkeiten bei verlangsamtem Sprechtempo. Diskriminierungen und Stigmatisierungen durch das soziale Umfeld sind mögliche Folgen.

Von besonderer Bedeutung ist auch das Phänomen, dass die personale Identifizierung in den beschriebenen Fällen durch eine nosologische abgelöst wird, d. h. die Person nicht mehr als Individuum, sondern als Zugehöriger einer bestimmten Erkrankungsgruppe identifiziert wird. In diesem Fall findet folglich eine Verschiebung der Sprecheridentität zur Erkrankungsgruppenidentität statt. Während also die Gruppenzuordnung beim gesunden Sprecher ein additives Merkmal zur eigentlichen Sprecheridentität darstellt, ersetzt sie bei bestimmten Erkrankungen die Sprecheridentität als das eigentliche Hauptmerkmal. Als charakteristisches Beispiel dieses Phänomens kann der erwachsene „Stotterer“ mit ausgeprägter Symptomatik genannt werden.

\section{Zum Begriff der Sprecheridentität}

Fragen zur Identität sind in der Philosophie spätestens seit der klassischen Antike - überliefert durch Heraklit, Plato, Aristoteles etc. - und in der Psychologie - spätestens seit Herbart, Fechner, Jung etc. - von Bedeutung. In den Sprachwissenschaften findet sich der Identitätsbegriff in sprachenvergleichenden Studien zur Sprachenentstehung und zur Ausprägung regionaler Dialekte. Innerhalb der Medizin ist eine fachliche Tradition des Identitätsbegriffes bisher besonders in den Bereichen Medizinpsychologie, Psychosomatik und Psychiatrie ${ }^{5}$ auszumachen. In der Phoniatrie, die sich wesentlich mit der Diagnostik und Behandlung monokausaler und komplexer Kommunikationsstörungen beschäftigt, ist die Bedeutung der personalen Identität bzw. ihres

5 Huber (1999). 
Verlustes für die unterschiedlichsten Stimm-, Sprech- und Sprachstörungen zwar seit der Fachgründung durch Gutzmann $1905^{6}$ implizit durchgängig dokumentiert. ${ }^{7}$ Es finden sich in der Literatur bisher aber kaum Studien, welche die Grundbedingungen der Sprecheridentität kategorial bestimmen und deren expressive und perzeptive bzw. psychoakustische Bedeutung für Sprecher und Hörer qualitativ und quantitativ analysieren. Dies bedeutet zugleich, dass derzeit keine zuverlässige Aussage möglich ist hinsichtlich der Frage, wann die personale Identifizierung durch eine nosologische abgelöst wird, d. h. wann betroffene Sprecher als krank wahrgenommen werden bzw. „Normalität" einbüßen.

Philosophische Konzepte, die den Identitätsbegriff prozesshaft, d. h. im Sinne der Vermittlung eines Subjekts mit seiner gesellschaftlichen Umgebung verstehen, wie diejenigen von Mead $^{8}$ und Frey, ${ }^{9}$ und primär deren psychosozialen Kontext thematisieren, wie etwa Erikson, ${ }^{10}$ waren aus phoniatrischer Sicht bisher wenig hilfreich, obgleich auch in der Philosophie und Soziologie ${ }^{11}$ Kommunikation als notwendige Bedingung der Identitätsbildung angesehen wird. Eine mögliche Erklärung hierfür bilden die weitgehende methodische Vernachlässigung der empirischen Seite von Stimme und Sprechen in der philosophischen und bioethischen Forschung sowie die unzureichende kommunikationstheoretische Aufarbeitung der parametrisch fundierten Erkenntnisse innerhalb der Phoniatrie.

Empirische Erfahrungen mit parametrischen Techniken zur akustischen Stimm- und Sprachschallanalyse liegen in der Phoniatrie seit den 196oer Jahren vor. ${ }^{12}$ Diese Techniken werden beispielsweise eingesetzt, um aperiodische spektrale Störschallkomponenten bei der Stimmlippenschwingung (Dysphonie), ${ }^{13}$ pathologische Strömungsgeräusche bei der Artikulation (Dyslalie) und Reduktionen der allgemeinen Sprechverständlichkeit (Dysarthrie, Dysglossie, Rhinophonie $)^{14}$ qualitativ und quantitativ zu analysieren. Allerdings spiegeln die Befunde dieser schallanalytischen Verfahren erfahrungsgemäß weder das subjektiv erlebte Ausmaß der bestehenden Störung noch deren Auswirkungen auf die Lebensqualität der Erkrankten wider. Auch ist es bisher nur ansatzweise gelungen, untersucherunabhängig reproduzierbare Befundqualitäten im Bereich Stimme ${ }^{15}$ und Sprechen ${ }^{16} \mathrm{zu}$ erarbeiten, auf deren Grundlage sich kohärente Schweregradabstufungen aufbauen ließen. Es ist zu vermuten, dass diese Schwierigkeiten darauf zurückzuführen sind, dass den bisherigen empirischen Verfahren ein die Persönlichkeit erfassen-

\footnotetext{
6 Das erste Fachlehrbuch erschien 1909, vgl. Gutzmann (1909), S. 1-208.

7 Vgl. Wendler et al. (1996) und Böhme (1997).

8 Mead (2005).

9 Frey (1987).

10 Vgl. Übersicht bei Noack (2005).

11 Vgl. Krappmann (2005).

12 Vgl. hierzu Klingholz (1986) und Schultz-Coulon/Klingholz (1988).

13 Yanagihara (1967a, b); Fröhlich et al. (1998).

14 Darley et al. (1969a, b), Weismer et al. (2001).

15 Bergan/Titze (2001), Wuyts et al. (2000).

16 Zyski/Weisiger (1987).
} 
des, übergreifendes Grundkonzept fehlt. Hier bietet sich ein interdisziplinär orientiertes Konzept zur Sprecheridentität als Bezugsgröße an.

Während die Phoniatrie - und damit die betroffenen, von Diskriminierung und Ausgrenzung bedrohten Patienten - von einer übergreifenden Konzeption philosophischer, kommunikationstheoretischer oder medizinethischer Begrifflichkeit profitieren würde, könnten die philosophisch orientierten Fächer so einen neuartigen parametrisch-modularen Zugang zum Identitätsbegriff erhalten.

\section{Sprecheridentität als Resultat der physiologischen Stimm- und Sprechfunktion}

In dem bei der verbalen Kommunikation übermittelten Sprachsignal verbinden sich die stimmlichen, sprecherischen und sprachlichen Merkmale einer Person zu einem komplexen Gebilde, das sich als individuelle Sprecheridentität bezeichnen lässt. Diesen Merkmalen liegen die im Folgenden beschriebenen Entstehungsmechanismen zugrunde.

Grundlage der verbalen Kommunikation ist die Erzeugung von Stimme als Trägersignal, das während des Sprechvorgangs sprachlich kodiert wird. Die Stimmerzeugung wird klassischerweise als triadisches System verstanden, dessen einzelne Komponenten sich während der Sprachschallerzeugung gegenseitig beeinflussen. Als Ausgangsenergie dient die Exspirationsluft, wobei die phonatorische Atemfunktion häufig mit der eines Windkessels verglichen wird. ${ }^{17}$ Als zweite Komponente des triadischen Systems wird die myoelastischaerodynamische Schwingungsfunktion der Stimmlippen bezeichnet, die als Tongenerator fungierend, die Luftsäule im angrenzenden Hohlraumsystem in Schwingungen versetzt. Dieses Hohlraumsystem wird als Ansatzrohr oder Vokaltrak $\mathrm{t}^{18}$ bezeichnet und bildet als dritte Komponente den Resonator, in dem sowohl die stimmliche Klanggestaltung als auch die sprecherische Lautkodierung vollzogen wird. Es schließt sich mundwärts an die Stimmlippen des Kehlkopfes an und wird durch Rachen, Zunge, Velum, Gaumen, Lippen und Nase begrenzt.

Die Sprechstimmlage resultiert aus der Stimmlippenlänge und -masse und aus der Grundspannung der Stimmlippen während ihrer Schwingung. Die Ansatzrohrmorphologie prägt grundlegende Klangeigenschaften der Stimme, die sich jedoch bei entsprechender Begabung in weiten Grenzen sängerisch und sprecherisch trainieren lassen. Abgesehen von diskreten geschlechtsdifferenten Lauteigenschaften, die sich aus der geschlechtsabhängig unterschiedlichen Ansatzrohrlänge und -proportionalität ergeben, ${ }^{19}$ ist jede Person unabhängig von ihrer genetisch-rassischen Zugehörigkeit zum Zeitpunkt der Geburt fähig, die Lautproduktion jeder Weltsprache zu erlernen, d. h. jede sprachenbezogene und dialektale Sprecheridentität anzunehmen.

17 Vgl. Barth (1911), S. 56.

$18 \mathrm{Vgl}$. Fitch/Giedd (1999).

19 Vgl. Sundberg (1987), S. 114. 
Dem triadischen Stimm- und Sprechorgan übergeordnet sind die kortikalen Sprachzentren und deren Bahnsysteme, mit denen verbale Äußerungen geplant, initiiert und gesteuert werden, sowie die Hirnnervenkerngebiete, über die wesentliche phonatorische und artikulatorische Bewegungsabläufe vollzogen werden. Dem Hörorgan und dessen zentralnervösen Repräsentationen wird eine Feedback-Kontrollfunktion zugeschrieben, der im Sinne erlernter und habitueller Wahrnehmungsprozesse allgemein eine sprecheridentitätserhaltende Funktion zukommen soll.

Auf der Grundlage dieser physiologischen Stimm-, Sprech- und Sprachfunktionen wird den folgenden Merkmalen eine Bedeutung für die Charakteristik der Sprecheridentität zugeschrieben: Der als mittlere Sprechstimmlage bezeichneten überwiegenden Tonhöhe der Stimme in der Spontansprache kommt wesentliche Bedeutung für die Geschlechtszuschreibung und Alterseinschätzung eines Sprechers zu.

Die melodische Variation dieser Sprechstimmlage gemeinsam mit der Betonung und Pausengestaltung im Sprechablauf unterliegt zwar auch (vermutlich kulturell geprägten) Geschlechtseinflüssen der Person, ist aber auch Ausdruck emotionaler Crundstimmung und persönlichen Charakters. Als weitere Merkmale spezifischer Sprechereigenschaften sind u. a. Stimmstärke, Klangreinheit der Stimme, Sprechtempo, Lautcharakteristik, Artikulationsschärfe und Nasalität zu nennen. Besonderheiten der Themen- und Wortwahl sowie deren phrasischer und gestischer Umsetzung sind eher Gegenstand soziologischer, psychologischer und psychiatrischer Forschung, während sich die phoniatrische Forschung im Bereich der Sprecheridentität stärker auf die phonatorische, sprechmotorische und sprachsystematische Ausführung fokussiert.

\section{Störungen der Sprecheridentität: Eine Bestandsaufnahme}

\subsection{Störungen im Hinblick auf die Geschlechtszugehörigkeit}

Störungen der Sprecheridentität im Hinblick auf die Geschlechtszugehörigkeit können im Wesentlichen stimmlichen Eigenschaften zugeordnet werden. ${ }^{20}$ Die stimmlich verursachte geschlechtliche Fehlzuweisung von Jugendlichen und Erwachsenen wird häufig als äußerst diskriminierend erlebt. Besonders im beruflichen Telefonkontakt sind Diskrepanzen zwischen dem erwarteten Geschlecht und einer hierzu widersprüchlichen Kommunikationssituation nicht selten auch von monetärer Bedeutung.

Im Unterschied zur Mehrzahl der in den folgenden Abschnitten genannten Störungsbilder ist die Bedeutung der geschlechtsdifferenten stimmlichen Charakteristik in ihrer Bedeutung für die Sprecheridentität als solche in der Phoniatrie allgemein etabliert. Physiologischerweise verläuft die geschlechtsabhängige Stimmentwicklung vom Säugling zum alternden Menschen ausgehend von einer nahezu identischen stimmlichen Charakteristik zum Zeitpunkt der Geburt zunächst divergierend bis zur Ausprägung einer typischen Männerund Frauenstimme im Erwachsenenalter, wobei sich beide Geschlechter im

20 Vgl. hierzu die Untersuchung von Van Gemmeren (2003). 
Senium im Hinblick auf ihre typischen stimmlichen Eigenschaften konvergierend annähern. Störungen der stimmlichen Realisation der Geschlechtszugehörigkeit können angeboren oder erworben sein. Im ersteren Fall sind sie entweder genetisch-syndromal verursacht oder durch angeborene laryngeale Missbildungen. Die erworbenen Störungen lassen sich drei wesentlichen Ausprägungsformen zuordnen. So treten Störungen einerseits dadurch auf, dass Entwicklungsphasen des physiologischen Stimmwechsels (Mutation) nicht oder nur unzureichend vollzogen werden. Zweitens können sie durch krankheitsbedingte oder iatrogene Hormoneinflüsse ${ }^{21}$ verursacht werden. Im dritten Fall, dem der Transsexualität, stimmen die psychosoziale Selbstdefinition und das biologische Ursprungsgeschlecht nicht überein, so dass sekundär der Wunsch einer Stimmanpassung an das Zielgeschlecht entsteht. ${ }^{22}$

$\mathrm{Zu}$ den genetisch-syndromalen Stimmveränderungen mit fehlerhafter Ceschlechtsrepräsentation, in diesem Fall bei weiblichen Erkrankten, ist das Down-Syndrom als eines der am häufigsten vorkommenden Syndrome zu nennen, das mit einer laryngeal morphologisch-bedingt tiefen und rauen Stimme einhergeht, die eine weibliche Geschlechtsrepräsentation konterkariert. Beispiele für männliche Sprecheridentitätsstörungen bilden Syndrome mit fehlenden oder pathologisch additiven Geschlechts-Chromosomen, ${ }^{23}$ die eine physiologische Pubertät bzw. Mutation vereiteln.

Ein ausbleibender, unvollständiger oder verzögerter Stimmwechsel bei männlichen Jugendlichen hat nur sehr selten organische oder hormonelle Ursachen. In der Mehrzahl der Fälle handelt es sich um eine einfache und damit therapeutisch erfolgreich beeinflussbare Entwicklungsstörung. Neben intrafamiliärem Rollenverhalten insbesondere in der Mutter-Sohn-Interaktion oder bei fehlendem erwachsenen männlichen Vorbild werden auch Phänomene wie eine anhaltende Selbstidentifikation mit der „,kindlichen“ Stimme beispielsweise bei sehr erfolgreichen präpubertalen Chorsängern diskutiert.

Hormonelle Formen von Stimmstörungen bei Frauen, die mit einer erniedrigten Sprechstimmlage einhergehen, werden im Zusammenhang mit hormonellen Umstellungen im Verlauf von Schwangerschaft und Niederkunft berichtet. Je nach Ausprägung dieser Stimmveränderung wird diese von den Frauen selbst oder von Personen des näheren Umfeldes als Veränderung der Sprecheridentität gewertet. Eine besondere Ausprägung i. S. einer deutlichen Vermännlichung der Stimme findet man bei Erkrankungen, die mit einer pathologischen Freisetzung von Androgenen bei Frauen einhergehen. Bekanntestes Beispiel hierfür ist das Adrenogenitale Syndrom. ${ }^{24}$

Die gestörte Sprecheridentität bei männlichen und weiblichen Transsexuellen bzw. Transgendern bildet eine besondere Indikation zur phoniatrischen Intervention. Während Frau-zu-Mann-Transgender unter der hormonellen Umstellung im Rahmen des Geschlechtswechsels bereits ohne spezifische stimmtherapeutische Behandlungsverfahren zumindestens eine partielle ge-

21 Vgl. Heinemann (1976), S. 36-165.

$22 \mathrm{Vgl}$. Neuschaefer-Rube/Scheidt/Groß in diesem Band.

23 Z. B. Klinefelter-Syndrom.

24 Heinemann (1976), S. 133-143. 
schlechtsbezogene Stimmanpassung erfahren, suchen Mann-zu-Frau-Transgender regelmäßig um phoniatrische Unterstützung nach, da Ausgangsstimme und Zielstimme hinsichtlich ihrer mittleren Sprechstimmlage, ihrer prosodischen Eigenschaften und ihrer spezifischen durch den männlichen Vokaltrakt charakterisierten Formantgestaltung differieren.

\subsection{Verlust der Sprecheridentität nach Laryngektomie}

Bei ausgedehnten Kehlkopfkarzinomen ist die Totalentfernung des stimmerzeugenden Larynx auch bei Einsatz moderner HNO-chirurgischer Verfahren häufig noch die Therapie der ersten Wahl.

Kompensatorisch sind nach Laryngektomie je nach Ausgangssituation in den meisten Fällen drei Strategien zur Ersatzstimmbildung verfügbar: erstens die rehabilitative Anbahnung einer Ösophagusersatzstimme, zweitens die Stimmerzeugung unter Verwendung einer chirurgisch implantierten Stimmprothese und drittens die Stimmgebung unter Zuhilfenahme eines externen, am Hals platzierten Tongenerators wie z. B. eines Servox-Gerätes.

Die Ösophagusersatzstimme ist die klassische Methode der Ersatzstimme nach erfolgter Kehlkopfentfernung. Medizinisch wird sie synonym als Ructusstimme bezeichnet, umgangssprachlich als sogenannte Rülpssprache. Verschluckte bzw. von der Zunge stempelartig in den Ösophagus gedrückte Luft wird hierbei ruckartig freigesetzt, um verschiebliche Schleimhaut, meist in Höhe des Ösophagussphinkters (Pseudoglottis) in Schwingungen zu versetzen, wodurch die Luftsäule des Vokaltraktes angeregt wird. Die resultierende Stimme liegt bei männlichen und weiblichen Sprechern ähnlich tief, nämlich deutlich unterhalb der üblichen Stimmlage einer Männerstimme. Die Rülpscharakteristik überlagert die Ansatzrohr-bedingte Klang- und Lautcharakteristik des Sprechers. Hinzu kommen die akustischen Auswirkungen der morphologischen Ansatzrohrveränderungen, die sich durch die Pharynxverkürzung im Rahmen der Tumoroperation ergeben. Neben dem klanglichen Verlust der Sprecheridentität und neben der durch die geringe Luftmenge determinierten Phrasenverkürzung in der Spontansprache bewirkt die Rülpscharakteristik der Stimme in unserem kulturellen Umfeld eine zusätzliche Stigmatisierung, weckt sie doch unbewusste Aversionen beim Zuhörer gegenüber einer als unfein geltenden stimmlichen Äußerung.

Die Ersatzstimmbildung nach Einsetzen einer Stimmprothese ermöglicht längere Phrasenbildungen unter Erhalt von Betonungsmustern, da durch die prothesenbedingte tracheoösophageale Fistel die Exspirationsluft direkt zur Schwingungsanregung eingesetzt werden kann. Wenngleich auch bei diesem Stimmmechanismus eine Pseudoglottis als Tongenerator fungiert, so klingt die Stimme weniger tief und insgesamt deutlich physiologischer als die Ructusstimme ohne Prothese. Unter den Ersatzstimmformen nach Laryngektomie bleibt die Sprecheridentität bei dieser Stimmform am Besten erhalten.

Im Gegensatz hierzu ist die apparative Servoxstimme von metallisch roboterhaftem Klang und kann nur zwischen zwei Grundfrequenzen variiert werden. Alle Servoxsprecher klingen gleich und wecken beim Zuhörer Reminiszenzen an einschlägige Science-Fiction-Filme. Diese Ersatzstimmform 
wird von Personen mit regulärer Laryngektomie daher nur in ausgewählten Situationen und bei stimmlicher Ermüdung als ultima ratio eingesetzt. Eine Ausnahme bilden Karzinompatienten, bei denen aufgrund großer Tumorausdehnung der Ösophagussphinkter mitentfernt wurde. Diese Patienten können eine klassische Ructusstimme oft nicht ausbilden, wobei auch Stimmprothesenapplikationen nicht in allen Fällen möglich sind. Diese Sprecher sind demnach auf diese ungünstigste der Ersatzstimmformen angewiesen.

\subsection{Verlust der Sprecheridentität bei offener Rhinophonie}

Der Stimmklang bei offener Rhinophonie, ${ }^{25}$ umgangssprachlich als Näseln bezeichnet, entsteht dadurch, dass orale, d. h. typischerweise nicht-nasale Laute pathologischerweise durch fehlenden oder unvollständigen velopharyngealen Abschluss mit Nasenresonanz gebildet werden. Das bekannteste Beispiel bilden Personen mit plastisch nicht oder nur unvollständig verschlossenen Lippen-Kiefer-Gaumenspalten. In selteneren Fällen können klanglich vergleichbare Störungen auch im Rahmen von Gaumensegellähmungen oder bei habituellen Störungen der Gaumensegelfunktion vorkommen. Bei sämtlichen dieser Erkrankungen werden die Resonatoreigenschaften des Ansatzrohres so verändert, dass auch die Lautcharakteristik verändert wird, was sich mindernd auf die Sprechverständlichkeit der rhinophonischen Sprecher auswirkt. Klanglich dominiert die offene Rhinophonie die Sprechercharakteristik derart, dass Personen dieses Störungsbildes nicht mehr in ihrer personalen Besonderheit wahrgenommen werden, sondern kategorial in ihrer manchmal zutreffenden, manchmal vermeintlichen Zugehörigkeit zur Gruppe der Spaltenträger. Im Rahmen kriminaltechnisch durchgeführter stimmakustischer Analysen zur Identifikation von Straftätern bilden rhinophonisch erzeugte Sprachsequenzen aufgrund der sehr stark veränderten Ansatzrohrcharakteristik besondere Schwierigkeiten. Die akustische Charakteristik der offenen Rhinophonie mit der ihr eigenen Penetranz gibt oft Anlass, diese Gruppe Sprechgestörter kabarettistisch zu vereinnahmen. Im Sozialkontakt der Betroffenen untereinander geht der verbalen Kommunikation oft eine Verifizierung der Erkrankung des Gegenübers voraus, um für sich auszuschließen, dass der Andere die eigene Störung lediglich provozierend imitiert.

\subsection{Dysphonie}

Im Unterschied zu den bisher genannten Störungsbildern, die sowohl bezogen auf die Selbstwahrnehmung als auch bezogen auf die Fremdwahrnehmung die Sprecheridentität empfindlich beeinträchtigen, besteht in unserem kulturellen Umfeld eine erstaunliche Unempfindlichkeit gegenüber den verschiedenen Ausprägungsgraden von Heiserkeit, seien sie organisch oder auch funktionell verursacht. Zwar lässt sich objektiv nachweisen, dass der erhöhte Rauschanteil im Sprachschall, mit dem diese Störungen einhergehen, ${ }^{26}$

25 Auch als Rhinophonia aperta bezeichnet.

26 Vgl. Michaelis/Fröhlich/Strube (1998). 
die identitätsprägende Sprechercharakteristik erheblich überdeckt und auch Reduktionen der Sprechverständlichkeit verursachen kann. Eine Stigmatisierung der Betroffenen scheint daraus jedoch nicht zu resultieren. Es bleibt zu spekulieren, ob dieser Sachverhalt dadurch begründet ist, dass die Mehrheit der Erwachsenen unseres Kulturkreises im Rahmen typischer Infekte der oberen Luftwege auf eigene Erfahrungen mit dem Symptom Heiserkeit zurückblicken kann.

\subsection{Verlust der Sprecheridentität bei Redeflussstörungen}

Redeflussstörungen wie Stottern und Poltern zeigen Veränderungen der Kommunikationsfähigkeit besonders im Bereich der Sprechabläufe. Die Stimmgebung erfolgt zwar häufig verspannt, ist aber vergleichsweise geringer beeinträchtigt als die Lautbildung und das Redefluss-Pausenverhalten im Sprechakt. Besonders stimmlose Konsonanten, speziell im Anlaut wirken als Stopper im Fluss der artikulatorischen Bewegungen, was sich derart auswirken kann, dass bestimmte Teile des muttersprachlichen und fremdsprachlichen Wortschatzes vollständig vermieden werden.

Hinsichtlich der Selbstwahrnehmung des veränderten Sprechverhaltens sind Personen mit Stottersyndrom häufig äußerst kritisch bis hin zu einem generalisierten sprachlichen Vermeidungsverhalten oder dem Aussetzen stressbehafteter und problematischer Situationen. Personen mit Poltersyndrom zeigen dagegen häufig eine selbstbewusste Persönlichkeit bis hin zur teilweisen oder vollständigen Negierung der vorhandenen Redeflusssymptomatik.

Da sich Stottern und Poltern überwiegend im Kindesalter ausbilden, ${ }^{27}$ ist die Zuschreibung eines Verlustes der Sprecheridentität missverständlich, da sich eine symptomfreie Sprecheridentität während des Spracherwerbs und des erlernten Rollenverhaltens häufig gar nicht erst ausbilden kann. Stotterer bilden auch die Form der Kommunikationsstörung aus, die in der Öffentlichkeit individuell und in kulturellen Veranstaltungen am Häufigsten imitiert wird. Diese Störungsform wird in ganz besonderem Maße durch eine nosologisch definierte „Stottereridentität“ geprägt, die die physiologischerweise vorhandene personale Sprecheridentität der Erkrankten substituiert.

\subsection{Verlust der Sprecheridentität bei Sprech- und Sprachstörungen}

Dyslalie: Die Dyslalie wird je nach klinischem Erscheinungsbild, das eher phonetisch oder eher phonematisch charakterisiert ist, als isolierte Artikulationsstörung oder als Teil einer sprachsystematischen Störung aufgefasst. Umgangssprachlich als „Stammeln“ bezeichnet, ist dieses häufig durch ein pathologisches Strömungsgeräusch gekennzeichnet, dass die Lautbildung charakteristisch modifiziert. Seine häufigste Form, die eines interdentalen, seltener addentalen Sigmatismus (Lispeln) wird ihrer Häufigkeit zufolge eher

27 Sehr häufig tritt die Redeflussstörung zwischen dem 2.-5. Lebensjahr auf, in $98 \%$ vor dem 10. Lebensjahr. Vgl. hierzu Böhme (1997), S. 83. 
als kosmetische Normdeviation denn als krankhaft abweichende Sprechercharakteristik aufgefasst.

Dysglossie, Sprechapraxie und Dysarthrie: Sprechmotorische Störungen können einerseits daraus resultieren, dass die am Bewegungsvorgang beteiligten Artikulatoren und hier insbesondere die Zunge und/oder das Gaumensegel, d. h. die peripheren Organe, durch entzündliche, narbige oder tumoröse Einflüsse raumfordernd beeinträchtigt werden oder dadurch, dass Teile dieser Artikulatoren aufgrund vorangegangener chirurgischer Behandlungsmaßnahmen entfernt wurden (Dysglossie). Sprechmotorische Störungen können andererseits dadurch entstehen, dass Erkrankungen im zentralen Nervensystem, eine Störung der artikulatorischen Planung und Steuerung von Sprechbewegungen verursachen (Sprechapraxie) oder wesentlich häufiger die Bewegungsausführung selbst beeinträchtigen (Dysarthrie). Bei allen genannten Erkrankungen zeigen sich Veränderungen im Bereich der Sprechgeschwindigkeit, der Artikulationsschärfe und damit auch der erzeugten Sprechverständlichkeit der Erkrankten. ${ }^{28}$ Die persönlichen identitätseigenen Sprechercharakteristika werden durch die verschliffene Artikulation und bei Dysarthrien häufig auch durch eine zusätzlich bestehende offene Rhinophonie überlagert. Aufgrund der auditiven Ähnlichkeit besonders dysglossischer und dysarthrischer Störungen mit Zuständen von Sprechern im voll alkoholisierten Zustand werden Personen dieser Erkrankungsgruppen von Fremden nicht selten letzterem Personenkreis zugeordnet, mit sämtlichen Zuschreibungen deklassierender Art. Insbesondere Schuldzuweisungen hinsichtlich fehlenden Verantwortungsbewusstseins und mangelnder Willenskraft wirken sich nicht selten diskriminierend aus.

Aphasie: Während sich die im letzten Abschnitt beschriebenen Einflüsse der Erkrankungen auf die sprechmotorische Realisation kognitiv frei entwickelter Sprachäußerungen beziehen, sind spezielle Formen einer Aphasie zusätzlich dadurch gekennzeichnet, dass der Zugriff auf Wortschatz und Syntax bereits gedanklich gestört sein kann und selbst in Form der sprachlichen Vorstellungswelt nicht mehr verfügbar ist. Diese Störungen betreffen den elementaren Kern jeder Persönlichkeit, bereits vor jeder Ausführung des Sprechaktes selbst. Im Titel des Klassikers von Oliver Sacks: „Der Mann, der seine Frau mit seinem Hut verwechselte“ findet sie ihren oft zitierten medizinisch-literarischen Ausdruck. Bei den verschiedenen Formen von Aphasie handelt es sich daher um die schwerwiegendste Form gestörter Sprecheridentität im Rahmen vorhandener Kommunikationsstörungen.

\subsection{Verlust der Sprecheridentität im Zusammenhang mit Störungen des Hörvermögens}

Spätertaubte Jugendliche und Erwachsene haben einen teilweise oder vollständig auditiv kontrollierten Spracherwerb vollzogen. Je nach Ausmaß der vorhandenen Hörstörung und je nach Zeitpunkt und Qualität der apparativen Versorgung mit Hörhilfen ist ihre sprechmotorische Kommunikationsfähig-

$28 \mathrm{Vgl.} \mathrm{Kent/Kim} \mathrm{(2003).}$ 
keit so wie die Hörgesunder oder hinsichtlich von Sprechgeschwindigkeit, ${ }^{29}$ Tonhöhenmodulation und Artikulationsschärfe charakteristisch verändert, so dass zumindest Kommunikationspartner, geübt im Umgang mit diesem Personenkreis, charakteristische, für Hörgestörte typische Sprech- und Stimmauffälligkeiten wahrnehmen. Bei zuvor physiologischem verbalen und artikulatorischen Sprechverhalten tritt bei plötzlicher Ertaubung einer Person eine als Sprachzerfall charakteristierte Kommunikationsstörung auf, die für Angehörige des persönlichen Umfeldes in Form eines veränderten Sprechverhaltens bemerkt wird. Da simultan der perzeptive Verlust für die Ertaubten die verbale Kommunikationsfähigkeit als solche verhindert, sind die Bedeutung des veränderten aktiven Sprechverhaltens und die damit veränderte Sprecheridentität für diese Personen zunächst von nebenrangiger Bedeutung.

\section{Zwischenbilanz und Ausblick}

Die Ausbildung von (individueller) Identität ist abhängig von sozialen Interaktionen eines Individuums mit anderen Menschen. Identität wird maßgeblich über Sprache und Sprechen vermittelt: $:^{30}$ Treffen Gesprächspartner aufeinander, so tauschen sie mittels Sprache, Gestik und/oder Mimik Absichten, Wünsche und Bedürfnisse aus. Ein Mensch verliert dann seine Identität, wenn er sich so verändert, dass wesentliche Kriterien, anhand derer er identifiziert wird, oder wesentliche Instanzen, welche für den Prozess der Identifizierung maßgeblich sind, entfallen oder geändert werden. Ein mögliches (überdies medizinisch relevantes) Beispiel hierfür ist der Verlust der Sprecheridentität.

Es war Zielsetzung des vorliegenden Beitrags, aus phoniatrischer Sicht das breite Spektrum möglicher Ursachen eines Verlustes von Sprecheridentität aufzuzeigen und somit die medizinische Relevanz dieses Problems zu verdeutlichen. Gleichzeitig galt es, auf die unseres Erachtens bisher wenig beachtete Tatsache hinzuweisen, dass dieser Identitätsverlust für den Betroffenen über die medizinisch-klinische Manifestation hinaus erhebliche psychosoziale Auswirkungen haben kann, die sich in Form von Diskriminierung oder Stigmatisierung äußern und zu Rückzugstendenzen der Betroffenen führen können - etwa dann, wenn eine bestehende Dysarthrie als Sprechen im alkoholisierten Zustand fehlinterpretiert wird. Der Verlust von Sprecheridentität ist damit zugleich ein Verlust von „Normalität“ bzw. die Ursache dafür, dass der Patient als „anders“ wahrgenommen wird.

Der Begriff Sprecheridentität, das damit angesprochene Phänomen und die mit dem Identitätsverlust einhergehende psychosoziale Problematik finden in der medizinischen Praxis noch wenig Aufmerksamkeit. Aus diesem Grund fehlen bisher weitgehend die Kriterien, um therapeutisch zu beurteilen, wie der einzelne Patient den teilweisen oder vollständigen Verlust seiner Fähigkeit zur sprachlichen Informationsübertragung einerseits und den Verlust seiner Sprecheridentität andererseits einschätzt. Für die Zukunft bietet sich dem-

29 Vgl. hierzu die Diplomstudie von Möldner (2006).

30 Vgl. Krappmann (2005). 
entsprechend ein reiches Betätigungsfeld, dessen Bedeutung weit über die Entwicklung klinisch-phoniatrischer Konzepte hinausweist.

\section{Literatur}

Baken/Orlikoff (2000): Ronald J. Baken, Robert F. Orlikoff, Clinical Measurement of Speech and Voice, San Diego 2000

Barth (1911): Ernst Barth, Physiologie, Pathologie und Hygiene der menschlichen Stimme, Leipzig, 1911

Bergan/Titze (2001): Christine C. Bergan, Ingo R. Titze, Perception of Pitch and roughness in vocal signals with subharmonics, Journal of Voice 15 (2001), 2, p. 165-175

Böhme (1997): Gerhard Böhme, Sprach-, Sprech-, Stimm- und Schluckstörungen. Ein Lehrbuch, Stuttgart 1997, S. 83

Darley/Aronson/Brown (1969a): Frederic L. Darley, Arnold E. Aronson, Joe R. Brown, Differential diagnostic patterns of dysarthria, Journal of Speech and Hearing Research 12 (1969), p. 246-269

Darley/Aronson/Brown (1969 b): Frederic L. Darley, Arnold E. Aronson, Joe R. Brown, Clusters of deviant speech dimensions in the dysarthrias, Journal of Speech and Hearing Research 12 (1969), p. 462-496

Fitch/Giedd (1999): W. Tecumseh Fitch, Jay Giedd, Morphology and development of the human vocal tract. A study using magnetic resonance imaging, Journal of the Acoustical Society of America 106 (1999), 3, p. $1511-1522$

Frey (1987): Hans-Peter Frey (Hrsg.), Identität. Entwicklungen psychologischer und soziologischer Forschung, Stuttgart 1987

Fröhlich/Michaelis/Kruse (1998): M. Fröhlich, D. Michaelis, Eberhard Kruse, Objektive Beschreibung der Stimmgüte unter Verwendung des Heiserkeits-Diagramms, HNO 46 (1998), 7, S. 684-689

Gemmeren (2003): Sabine van Gemmeren, Stimmig?! Geschlechtsspezifische Merkmale der Stimme und ihre Abweichungen, Diplomarbeit, Heilpädagogische Fakultät zu Köln in Kooperation mit der Phoniatrie Aachen 2003

Gutzmann (1909): Hermann Gutzmann, Physiologie der Stimme und Sprache, Braunschweig 1909

Heinemann (1976): Manfred Heinemann, Hormone und Stimme, Leipzig 1976

Huber (1999): Gerd Huber, Psychiatrie. Lehrbuch für Studium und Weiterbildung, Stuttgart 1999

Kent/Kim (2003): Ray D. Kent, Yunjung Kim, Toward an acoustic typology of motor speech disorders, Clinical Linguistics \& Phonetics 17 (2003), 6, p. 427-445

Klingholz (1986): Fritz Klingholz, Die Akustik der gestörten Stimme, Stuttgart 1986, S. 1-90

Krappmann (2005): Lothar Krappmann, Soziologische Dimensionen der Identität, Stuttgart 2005

Mead (2005): George Herbert Mead, Geist, Identität und Gesellschaft, Frankfurt a. M. 2005

Michaelis/Fröhlich/Strube (1998): Dirk Michaelis, Matthias Fröhlich, Hans Werner Strube, Selection and combination of acoustic features for the description of pathologic voices, Journal of the Acoustical Society of America 103 (1998), p. 1628-1639

Möldner (2006): Kristin Möldner, Vergleich der Sprech- und Stimmdynamik hörgeschädigter und normalhörender Jungen. Diplomarbeit der Lehr- und Forschungslogopädie, erstellt an der Klinik für Phoniatrie, Pädaudiologie und Kommunikationsstörungen, Aachen 2006

Neuschaefer-Rube/Spiecker-Henke (2002): Christiane Neuschaefer-Rube, Marianne Spiecker-Henke, Diagnostik funktioneller und organischer Stimmstörungen, in: Manfred Grohnfeldt (Hrsg.), Lehrbuch der Sprachheilpädagogik und Logopädie, Band 3, Diagnostik, Prävention und Evaluation, Stuttgart 2002, S. 283-303

Neuschaefer-Rube/Scheidt/Groß (2008): Christiane Neuschaefer-Rube, David D. I. Sander Scheidt, Dominik Groß, Modelle zur Definition von Transsexualität und ihre Auswirkungen auf die gesellschaftliche Akzeptanz - Das Beispiel Stimme und Sprechverhalten, in diesem Band

Noack (2005): Juliane Noack, Erik H. Eriksons Identitätstheorie, Oberhausen 2005

Sacks (1990): Oliver Sacks, Der Mann, der seine Frau mit seinem Hut verwechselte, Reinbek 1990

Scheidt (2003): David D. J. Sander Scheidt, Entwicklung und Erprobung eines Diagnostikkonzeptes für die logopädische Stimmarbeit mit Transgenders am Beispiel der sogenannten Frau-zu-Mann-Transsexualität. Diplomarbeit im Studiengang Lehr- und Forschungslogopädie, RWTH Aachen 2003 
Schultz-Coulon/Klingholz (1988): Hans-|ürgen Schultz-Coulon, Fritz Klingholz, Objektive und semiobjektive Untersuchungsmethoden der Stimme, in: Gerhard Kittel, Bernd Schürenberg (Hrsg.), Objektive und semiobjektive Untersuchungsmöglichkeiten von Stimme, Sprache und Gehör, Köln 1988

Spiecker-Henke (1997): Marianne Spiecker-Henke, Leitlinien der Stimmtherapie, Stuttgart 1997

Stengel/Strauch (1996): Ingeborg Stengel, Theo Strauch, Stimme und Person, Stuttgart 1996

Sundberg (1987): Johan Sundberg, The Science of the singing Voice, Dekalb 1987

Titze (1989): Ingo R. Titze, Physiologic and acoustic differences between male and female voices, Journal of the Acoustical Society of America 85 (1989), 4, p. 1699-1707

Weismer et al. (2001): Gary Weismer, ling-Yi Jeng, Jacqueline S. Laures et al., Acoustic and intelligibility characteristics of sentence production in neurogenic speech disorders, Folia Phoniatrica et Logopedica 53 (2001), p. 1-18

Wendler et al. (1996): Jürgen Wendler, Wolfram Seidner, Gerhard Kittel et al., Lehrbuch der Phoniatrie und Pädaudiologie, Stuttgart 1996, S. 44-132

Wuyts et al. (2000): Floris L. Wuyts, Marc S. De Bodt, Gert Moolenberghs et al., The dysphonia severity index: An objective measure of vocal quality based on a multiparameter approach, Journal of Speech, Language and Hearing Research 43 (2000), p. 796-809

Yanagihara (1967a): N. Yanagihara, Significance of harmonic changes and noise components in hoarseness, Journal of Speech and Hearing Research 10 (1967), p. 531-541

Yanagihara (1967b): N. Yanagihara, Hoarseness: Investigation of the physiological mechanisms, Annals in Otology 76 (1967), p. 472-489

Zyski/Weisiger (1987): B. J. Zyski, B. E. Weisiger, Identification of dysarthria types based on perceptual analysis, Journal of Communication Disorders 20 (1987), p. 367-378 